\title{
Nanophotonics
}

SPIEDigitalLibrary.org/jnp

\section{Molecular beacons involving highly luminescent colloidal quantum dots}

\author{
Mikhail Artemyev \\ Igor Nabiev
}




\title{
Molecular beacons involving highly luminescent colloidal quantum dots
}

\author{
Mikhail Artemyev ${ }^{\mathrm{a}, \mathrm{b}}$ and Igor Nabiev ${ }^{\mathrm{b}, \mathrm{c}}$ \\ ${ }^{a}$ Belarusian State University, Institute for Physico-Chemical Problems, 220030 Minsk, Belarus \\ m_artemyev@yahoo.com \\ ${ }^{\mathrm{b}}$ Moscow Engineering Physics Institute, Laboratory of Nano-Bioengineering, 31 Kashirskoe sh., \\ 115409 Moscow, Russian Federation \\ 'Institute of Molecular Medicine, European Technological Platform Semiconductor \\ Nanocrystals, Trinity College Dublin, James's Street, Dublin 8, Ireland
}

Paper 12132CT received Sep. 24, 2012; revised manuscript received Sep. 26, 2012; accepted for publication Sep. 28, 2012; published online Oct. 24, 2012.

Molecular beacons are fluorescently labeled biological systems, such as sequence-specific oligonucleotide hairpin structures with a stem and a loop, Eclipse probes, and nonnucleotide protein-based structures that react to ligand binding by generating a strongly enhanced fluorescent signal. For example, in oligonucleotide hairpin structures with a stem and a loop, a fluorescent probe (emitter) is chemically attached to one end of the loop structure, and a fluorescent quencher is attached to the other end. ${ }^{1}$ In the off-state, the fluorescent emitter is in close contact with the quencher, and luminescence is absent due to the Förster resonance energy transfer (FRET) or another type of energy transfer (ET) from the emitter to the quencher. During the detection procedure, the molecular beacon binds to its target, the complementary oligonucleotide sequence, opening the hairpin structure and spatially separating the emitter and the quencher, thus enabling luminescence. The detection scheme may be different if it is based on single-color fluorescence quenching or fluorescence release upon targeted binding to the analyte or spectrally encoded fluorescence signal, when both the FRET donor and the FRET acceptor are fluorescence probes capable of emitting. However, the main principle of the beacon operation is always the same and is based on detection of the changes in FRET efficiency between the emitter and the quencher via controllable spatial separation between the FRET donor and acceptor.

Generally, both FRET donors and FRET acceptors are organic dye molecules specifically selected to achieve a strong spectral overlap between the donor emission band and the acceptor optical absorption, which is necessary for efficient FRET. Instead of dyes, ultrasmall metal (gold, silver) nanoparticles are widely used as FRET quenchers due to highly efficient ET to their surface plasmons. Recently, highly luminescent semiconductor nanocrystals prepared using wet chemistry methods [colloidal quantum dots (QDs)] were employed for engineering molecular beacons ${ }^{2}$ and other FRET-employing inorganic/organic hybrid constructions and schemes (Fig. 1) ${ }^{3-5}$ The advantage of colloidal QDs over organic dyes exists in their remarkable optical properties. Like conventional bulk semiconductors, QDs absorb light in a broad spectral range above their band gap, which makes them almost ideal candidates for FRET acceptors. Moreover, the molar absorption coefficient of QDs may be an order of magnitude larger than those of organic dyes. The broad absorption spectrum means that QDs with different emission colors can be efficiently excited by a single source of light. In addition, the emission band of highquality colloidal QDs is much narrower than that of organic dyes (20 to $30 \mathrm{~nm}$ FWHM in QDs versus ca. $100 \mathrm{~nm}$ for dyes) and can be precisely tuned with nanometer accuracy over an extremely wide spectral range (ca. 300 to $3000 \mathrm{~nm}$ ) by merely changing the size and chemical composition of QDs during synthesis. This, together with an almost 1000-fold larger photostability as compared to dyes, makes QDs ideal candidates for molecular beacons. Unfortunately, ideal things are rare in nature.

As-synthesized green-emitting CdSe QDs ca. $2.5 \mathrm{~nm}$ in diameter are an order of magnitude larger and two orders of magnitude heavier than, e.g., fluorescein molecules. The same CdSe

0091-3286/2012/\$25.00 ㄷ 2012 SPIE 


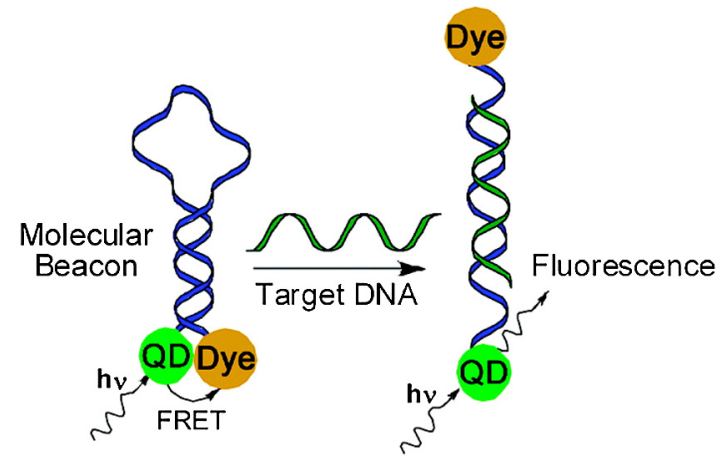

Fig. 1 Analysis of a target DNA by means of a hairpin nucleic acid probe functionalized with a CdSe/ZnS QD and a quencher. Reprinted with permission. ${ }^{5}$ Copyright 2011 American Chemical Society.

QDs water-solubilized by capping them with a shell of hydrophilic molecules have a hydrodynamic diameter no less than $8 \mathrm{~nm}$. Furthermore, water-soluble QDs usually carry a shell of charged molecules on their surface. The electrostatic interaction between charged groups on the surface of a QD and an oligonucleotide or protein is accompanied by formation of nanoparticles of large mass and hydrodynamic size, ${ }^{6}$ which makes precise control of a molecular beacon's on-off operation difficult. These problems mainly pertain to the physical chemistry of nanoparticles, organic molecules, and their assemblies. What are the main specific aspects of nanophotonics that are revealed in the operation of molecular beacons based on QDs?

When two dye molecules interact with each other via FRET in a molecular beacon, they can be treated as two point-like optical dipoles of equal sizes. This process can be described in terms of the classical Förster theory. The situation is much more complicated when either the emitter or the quencher or both are non-point-like nanosized particles, such as CdSe QDs. The excited electron-hole states in a QD are delocalized throughout the volume, and the corresponding optical dipole should be considered as an extended one (in contrast to a point-like optical dipole in a dye molecule). FRET between extended and point-like dipoles does not comply with the classical Förster rules. In fact, this results in a far less efficient FRET from the QD to the dye molecule in a QD-dye pair. ${ }^{5}$ The FRET efficiency lower than 50\% was obtained for a 1:1 QD-dye ensemble with a dye molecule chemically bound to the surface of a CdSe/ZnS QD via a short aminoethanethiol linker. ${ }^{7}$ The claimed $90 \%$ FRET quenching in molecular beacons with a CdSe QD donor and a dye acceptor seems to be a rare occasion. ${ }^{8}$ The situation is even worse in the case of CdSe nanorods (NRs) used as FRET donors due to strong distance-limited ETs from the extended optical dipole in a CdSe NR tens of nanometers in length to the point-like dipole of the dye molecule. ${ }^{9,10}$ A FRET efficiency as high as $100 \%$ can be achieved when the QD donor is in close contact with a few acceptor molecules, ${ }^{11}$ but this is not the case with traditional molecular beacons with their one-to-one ratio between the donor and acceptor.

Apparently, efficient FRET in a one-to-one molecular beacon structure can be achieved only when both the emitter and the quencher are of the same size, e.g., a green CdSe/ZnS QD as a FRET donor and a red QD as an acceptor. This is especially useful in the case of ratiometric sensing platforms where quantitative analysis is based on the intensity ratio between simultaneous green and red emissions. However, permanent background emission from the QD acceptor may be an interfering factor. Instead of a QD-based acceptor, silver or gold plasmonic nanoparticles can be efficiently used to quench the donor emission in the off-state. ${ }^{2}$ Unfortunately, unlike the optical absorption spectra of semiconductor QDs, those of Ag and Au nanoparticles smaller than $10 \mathrm{~nm}$ do not cover the entire spectral range, which is especially problematic for quenching the luminescence of donors emitting in the deep-red and NIR regions of the optical spectrum. In this case, one may use non-emitting core nanocrystals of narrow-gap semiconductors, such as $\mathrm{PbS}, \mathrm{PbSe}$, or even $\mathrm{CdSe}$, doped with certain ions $\left(\mathrm{Cu}^{2+}, \mathrm{Ag}^{+}\right.$, etc. $)$that can completely quench the photoluminescence of CdSe QDs due to the formation of nonradiative recombination traps inside the CdSe core.

In conclusion, molecular beacons based on highly luminescent QDs have obvious nanooptical advantages and may replace organic dyes from their numerous photonic applications. 
On the other hand, the complicated surface chemistry and large sizes of QDs hamper their immediate practical use and require additional studies to engineer optimal beacons and optimize their application setups. Let us see what the future holds.

\section{References}

1. "Introduction on Molecular Beacons," Public Health Research Institute, New Jersey Medical School-University of Medicine and Dentistry of New Jersey, (15 October 2012), http://www.molecular-beacons.com/MB_intro_full.html.

2. M. De, P. S. Ghosh, and V. M. Rotello, "Applications of nanoparticles in biology," $A d v$. Mater. 20(22), 4225-4241 (2008), http://dx.doi.org/10.1002/adma.v20:22.

3. I. Nabiev et al., "Fluorescent quantum dots as artificial antennas for enhanced light harvesting and energy transfer to photosynthetic reaction centers," Angew. Chem. Int. Ed. Engl. 49(40), 7217-7221 (2010), http://dx.doi.org/10.1002/anie.v49:40.

4. A. Rakovich et al., "Resonance energy transfer improves the biological function of bacteriorhodopsin within a hybrid material built from purple membranes and semiconductor quantum dots," Nano Lett. 10(7), 2640-2648 (2010), http://dx.doi.org/10.1021/nl1013772.

5. R. Freeman, B. Willner, and I. Willner, "Integrated biomolecule-quantum dot hybrid systems for bioanalytical applications," J. Phys. Chem. Lett. 2(20), 2667-2677 (2011), http:// dx.doi.org/10.1021/jz2006572.

6. B. J. Berne and R. Pecora, Dynamic Light Scattering with Applications to Biology, Chemistry, and Physics, Dover Publications, Mineola, NY (2000).

7. M. Artemyev, "Resonance energy transfer in conjugates of semiconductor nanocrystals and organic dye molecules," J. Nanophoton. 6(1), 061705 (2012), http://dx.doi.org/10.1117/1 .JNP.6.061705.

8. R. Freeman et al., "Optical aptasensors for the analysis of the vascular endothelial growth factor (VEGF)," Anal. Chem. 84(14), 6192-6198 (2012), http://dx.doi.org/10.1021/ ac3011473.

9. M. Hardzei et al., "Comparative efficiency of energy transfer from CdSe-ZnS quantum dots or nanorods to organic dye molecules," Chem. Phys. Chem. 13(1), 330-335 (2012), http:// dx.doi.org/10.1002/cphc.201100552.

10. M. Artemyev, E. Ustinovich, and I. Nabiev, "Efficiency of energy transfer from organic dye molecules to CdSe-ZnS nanocrystals: nanorods versus nanodots," J. Am. Chem. Soc. 131(23), 8061-8065 (2009), http://dx.doi.org/10.1021/ja809839h.

11. Y. Huang et al., "Intermolecular and intramolecular quencher based quantum dot nanoprobes for multiplexed detection of endonuclease activity and inhibition," Anal. Chem. 83(23), 8913-8918 (2011), http://dx.doi.org/10.1021/ac2013114. 